\title{
Frequency of Dopamine Concentration Transients Increases in Dorsal and Ventral Striatum of Male Rats during Introduction of Conspecifics
}

\author{
Donita L. Robinson, Michael L. A. V. Heien, and R. Mark Wightman \\ Department of Chemistry, Neuroscience Center, and Center for Alcohol Studies, University of North Carolina, Chapel Hill, \\ North Carolina 27599-3290
}

Transient, elevated concentrations of extracellular dopamine were characterized in the dorsal and ventral striatum of male rats during solitude, brief interaction with a conspecific, and copulation. Conspecific rats were systematically presented to male rats and allowed to interact for $30 \mathrm{sec}$; the males were kept in solitude between each presentation. During these episodes, 125 dopamine concentration transients from 17 rats were detected with fast-scan cyclic voltammetry at carbonfiber microelectrodes (peak amplitude, $210 \pm 10 \mathrm{~nm}$; duration, $530 \pm 20 \mathrm{msec}$ ). The frequency of dopamine transients increased sixfold during conspecific episodes compared with solitude. However, the phasic dopamine activity habituated on the second presentation of the conspecifics. When males were allowed to copulate with receptive females, additional dopamine transients were observed at frequencies $\sim 20 \%$ of those during the previous interaction episodes. A subset of these transients immediately preceded intromission. Overall, phasic dopamine activity appeared to be associated with input from multiple sensory modalities and was followed by a variety of approach and appetitive behaviors, consistent with electrophysiological observations of dopaminergic neuron burst-firing. In summary, (1) dopamine concentration transients occur in awake rats during solitude, in the absence of overt external cues; (2) dopamine transients are significantly more frequent in the presence of a conspecific, although this effect habituates; and (3) dopamine transients are less frequent during copulation than during brief conspecific episodes. These results establish for the first time that transient dopamine fluctuations occur throughout the dorsal and ventral striatum and demonstrate that they are more frequent with salient stimuli that elicit a response behavior.

Key words: dopamine; extracellular; solitude; social interaction; copulation; voltammetry
Considerable evidence indicates that dopamine systems in the forebrain contribute to various aspects of reinforced behavior (Wise and Rompre, 1989). Traditionally, nigrostriatal dopamine is viewed as necessary for sensorimotor coordination, and mesolimbic dopamine is viewed as necessary for appetitive and motivational aspects. Neurochemical measurements of dopamine, such as those provided by microdialysis, have supported these roles for dopamine during a variety of behaviors (Westerink, 1995). The time scale of these measurements (in minutes) provides an index of dopamine neurotransmission that arises from the normal, tonic firing of dopaminergic neurons and possibly a presynaptic modulation of release (Grace, 2000). However, dopaminergic neurons also fire more rapidly in phasic bursts of neuronal activity (Overton and Clark, 1997; Schultz, 1998). Such firing patterns should cause discrete chemical surges of dopamine into the extrasynaptic space, lasting for 1 or $2 \mathrm{sec}$ (Kawagoe et al., 1992; Suaud-Chagny et al., 1995). Only recently has technology

Received Aug. 7, 2002; revised Sept. 12, 2002; accepted Sept. 18, 2002.

This work was supported by National Institute of Neurological Disorders and Stroke Grant RO1 NS15841 (R.M.W.), National Institute on Drug Abuse Grant RO1 DA10900 (R.M.W.), and National Institute on Alcohol Abuse and Alcoholism Training Grant AA07573 (D.L.R.). We thank Megan Austin, Jessica Cantley, Lori Durham, Lisa Gurdin, Collin McKinney, and Sophia Papadeas for technical assistance, and Drs. Regina Carelli, Paul Garris, and Paul Phillips for helpful discussions. Chris Wiesen at the Odum Institute for Research in Social Science provided invaluable statistical guidance.

Correspondence should be addressed to Dr. R. Mark Wightman, Department of Chemistry, University of North Carolina, Chapel Hill, NC 27599-3290. E-mail: rmw@unc.edu.

Copyright (C) 2002 Society for Neuroscience $0270-6474 / 02 / 2210477-10 \$ 15.00 / 0$ advanced to the degree that such dopamine concentration transients, possibly arising from bursting of dopaminergic neurons, can be measured in behaving animals (Rebec et al., 1997; Robinson et al., 2001).

Rebec et al. (1997) measured dopamine concentration transients in the nucleus accumbens (NA) shell of male rats at their initial entrance to a novel environment. The chemical signal was not observed in the NA core, nor was it observed on subsequent reentry. We recently measured phasic dopamine signals in the context of sexual behavior (Robinson et al., 2001) and observed transient, robust increases in extracellular dopamine in the NA core of male rats on initial exposure to receptive female rats and subsequent approach behaviors. Both studies were remarkable in the linkage of discrete extrasynaptic dopamine signals with discrete salient events. However, nothing is yet known about the occurrence of dopamine transients in simple wakefulness or the relative frequency among striatal regions.

The purpose of the present study was to explore the incidence of dopamine concentration transients in the dorsal and ventral striatum in freely moving rats, replicating previous reports of transients in the NA, and extending these findings to the caudate putamen (CP) and olfactory tubercle (OT). We systematically compared phasic dopamine activity in the $\mathrm{CP}$, NA, and OT during solitude versus brief interaction with a conspecific, hypothesizing that the frequency of phasic dopamine would be higher in the presence of a conspecific, because it represents a biologically salient stimulus to the rat. We presented each conspecific (male, receptive female, and nonreceptive female) twice to evaluate 
whether the dopamine response habituated as predicted for the role of phasic dopamine as an alerting signal (Schultz, 1998; Redgrave et al., 1999). Finally, to replicate our previous findings in the NA and extend them to the CP and OT, we monitored extracellular dopamine during copulation in the same rats.

\section{MATERIALS AND METHODS}

Animals. Male and ovariectomized female adult Sprague Dawley rats (Charles Rivers, Raleigh, NC) were housed in a vivarium on a $12 \mathrm{hr}$ light/dark cycle (lights on at midnight) with food and water available $a d$ libitum. Males were singly housed; females were group-housed. All rats were well handled by the experimenter. Measurements were made in 17 male rats, which each received at least three 30 min opportunities for copulation before the experiment and reached ejaculation during at least two of these opportunities. Females were induced to behavioral estrus by the injection of $10 \mu \mathrm{g}$ of estradiol benzoate $48 \mathrm{hr}$ before and $500 \mu \mathrm{g}$ of progesterone $4 \mathrm{hr}$ before testing. They were checked for behavioral estrus by the presence of lordosis and proceptive behaviors (ear wiggling, hopping, and darting) when placed with a sexually experienced male before the experiment. All experimental procedures were approved by the Institutional Animal Care and Use Committee at the University of North Carolina.

Surgery. Surgical procedures were performed as described previously (Garris et al., 1997), with minor modifications. Male rats (350-450 gm) for electrochemical experiments were anesthetized with ketamine (80 $\mathrm{mg} / \mathrm{kg}$ ) and xylazine $(12 \mathrm{mg} / \mathrm{kg}$ ). A guide cannula (Bioanalytical Systems Inc., West Lafayette, IN), an $\mathrm{Ag} / \mathrm{AgCl}$ reference electrode, and a bipolar stimulating electrode (Plastics One, Roanoke, VA) were implanted in each rat as described previously (Robinson et al., 2001). The guide cannula was positioned $1.2 \mathrm{~mm}$ anterior and $2 \mathrm{~mm}$ lateral to bregma and extended $2.5 \mathrm{~mm}$ below the skull surface; all recordings were made on the right side of the brain. This placement allowed insertion of the carbon-fiber microelectrode into the CP (4-5.5 $\mathrm{mm}$ ventral from the skull surface), NA (6-8.4 mm ventral), or OT (8.8-9.2 mm ventral). The stimulating electrode was positioned at the medial forebrain bundle (4.1 $\mathrm{mm}$ posterior and $1.4 \mathrm{~mm}$ lateral to bregma; $\sim 8 \mathrm{~mm}$ ventral from the skull surface) or the substantia nigra/ventral tegmental area $(5.6 \mathrm{~mm}$ posterior and $1 \mathrm{~mm}$ lateral to bregma; $\sim 9 \mathrm{~mm}$ ventral from the skull surface) to stimulate dopaminergic fibers. The extracellular dopamine concentrations resulting from mild electrical stimulation of the dopamine pathway at these two placements did not differ, and were used as a calibration signal for each rat. Rats were allowed at least $2 \mathrm{~d}$ to recover after surgery.

Carbon-fiber microelectrode. Carbon-fiber electrodes were constructed using individual carbon fibers ( $6 \mu \mathrm{m}$ diameter, T650; Amoco, Greenville, SC) pulled and sealed in glass capillaries $(0.6 \mathrm{~mm}$ diameter $)$. The exposed fiber was trimmed to $130-225 \mu \mathrm{m}$ from the glass seal. The carbon fiber was acutely inserted with a detachable micromanipulator (Chemistry Instrument Facility, University of North Carolina) that locked into the guide cannula and allowed ventral placement of the electrode into the $\mathrm{CP}, \mathrm{NA}$, or OT at $<0.1 \mathrm{~mm}$ increments. The electrodes were calibrated for dopamine concentration after each experiment in an in vitro flow-cell system.

Voltammetric measurements. The carbon-fiber and reference electrodes were connected to a voltammetric amplifier mounted near the rat's head at the base of the tether. Voltammetric recordings were made at the carbon-fiber electrode every $100 \mathrm{msec}$ by applying a triangular waveform $(-0.4$ to $1.0 \mathrm{~V}$ vs the reference electrode, $300 \mathrm{~V} / \mathrm{sec})$ with an EI 400 potentiostat (Cypress Systems, Lawrence, KS). Each scan took $9.3 \mathrm{msec}$ to complete, and the electrode was held at $-0.4 \mathrm{~V}$ between scans. Voltammetric parameters, stimulation parameters, and data acquisition were controlled by a computer using LabVIEW computer instrumentation (National Instruments, Austin, TX) (Michael et al., 1999).

A high-throughput algorithm within the LabVIEW collection and analysis program was used to target potential dopamine concentration transients for additional investigation. A template of the subtracted cyclic voltammogram for dopamine for each particular animal was selected from the electrochemical response during the electrical stimulation of dopamine neurons. As a data file was loaded, the computer automatically scaled the current amplitude at the dopamine oxidation peak to the template, subtracting the average of 10 scans before the scan under evaluation. The mean squared error between the two voltammograms was calculated, the inverse of this quantity was plotted, and a cutoff point was determined, above which a voltammogram was targeted. To validate these targets as dopamine transients, the background-subtracted cyclic voltammograms at the targeted times were compared with those for dopamine. Criteria included peaks at approximately +0.6 and $-0.2 \mathrm{~V}$ versus $\mathrm{Ag} / \mathrm{AgCl}$, their relative amplitude, the absence of extraneous peaks, and a minimum of at least two consecutive scans. The duration of dopamine transients was determined from the cyclic voltammograms examined on a scan-by-scan basis. The peak amplitude of dopamine concentration, or $[\mathrm{DA}]_{\max }$, was measured from the current versus time plot at the dopamine oxidation potential and converted to concentration using postexperiment in vitro calibration of the carbon-fiber electrode.

Experimental design. Experiments were conducted during the animal's dark cycle, when the rats were most active. The test male was placed in a clean $60 \times 60 \mathrm{~cm}$ test chamber in a Faraday cage and attached to a tether secured to the stimulating electrode. The tether extended to a swivel at the top of the Faraday cage, allowing the rat full access to the test chamber. Rats were allowed at least $3 \mathrm{hr}$ to habituate to the cage and the tether on the experiment day. A new carbon-fiber microelectrode was then inserted into the guide cannula and lowered to the target region. The proximity to dopamine terminals was confirmed by the detection of electrically evoked dopamine release (24 rectangular pulses, $60 \mathrm{~Hz}, 120$ $\mu \mathrm{A}, 2 \mathrm{msec} /$ phase, biphasic).

Conspecific and solitary episodes. Conspecifics (receptive female, nonreceptive female, and male) were presented one at a time to the test male rat in random order and allowed to interact with the test rat for $30 \mathrm{sec}$. The conspecific rats were presented by gently pushing them over the lip of the test chamber and were manually removed after $30 \mathrm{sec}$. Conspecific episodes were defined as the $30 \mathrm{sec}$ interaction time plus the $10 \mathrm{sec}$ before and after presentation to account for any alerting stimuli (sounds, olfactory stimuli) associated with the introduction and removal of the conspecific. The brief time with the conspecific was designed to examine the effect of initial contact and yet militate against copulation, because 30 $\mathrm{sec}$ is generally shorter than the latency to intromit (Sachs and Barfield, 1976). The test animal was left undisturbed for an additional $190 \mathrm{sec}$ between presentations; these periods were termed solitary episodes. Dopamine concentration transients were continuously monitored throughout the solitary and conspecific episodes. Periodically, during solitary episodes, dopamine neurons were mildly stimulated (24 rectangular pulses, $60 \mathrm{~Hz}, 120 \mu \mathrm{A}, 2 \mathrm{msec} / \mathrm{phase}$, biphasic) to examine the electrode response. To test for the habituation of dopamine transients to the presentations, each conspecific was presented a second time, again in random order. Thus, each male received six brief conspecific presentations, interspersed with solitary episodes.

Copulation episode. After the conspecific and solitary episodes, the test rat was left undisturbed for $10 \mathrm{~min}$ before voltammetric data collection resumed. Then the receptive female was presented a third time and the pair was allowed to copulate. Electrochemical data were collected throughout the copulation period and for 2-4 min after ejaculation.

Behavioral analysis. The entire experiment was videotaped with a camera mounted in the Faraday cage. The electrochemical and behavioral data were synchronized with a video character generator (Chemistry Instrument Facility, University of North Carolina) that superimposed the episode and electrochemical scan numbers on the video record. Behavior for $\pm 5 \mathrm{sec}$ before and after each dopamine concentration transient was examined frame by frame and cataloged into $1 \mathrm{sec}$ bins, using the following categories: sniffing rat (whisker contact; sniffing face, body, anogenital region), general sniffing (ground or cage walls, air), orientation (head turn, whole body turn), locomotion to rat, and sexual behaviors (mounting, intromission, ejaculation, genital grooming). For statistical analysis, the total count for each behavior in the $5 \mathrm{sec}$ before a transient was compared with the count $5 \mathrm{sec}$ after the transient. In addition, copulatory behavior was evaluated by the construction of an event record of mounts (simple and ectopic), intromissions, ejaculations, genital grooming, and $22 \mathrm{kHz}$ vocalizations (Sachs and Barfield, 1976).

Histology. Rats were administered a lethal dose of urethane and perfused through the heart with saline and a $10 \%$ formalin solution. The brains were removed and stored in $4 \%$ paraformaldehyde in phosphate buffer until sliced into $40 \mu \mathrm{m}$ coronal sections and stained with thionine. The carbon fiber was too small to produce damage detectable with a light microscope. However, the dorsal part of the glass capillary left a detectable tract, and the total distance inserted was known from the micromanipulator, allowing reconstruction of the tip location.

Statistical analysis. Nonparametric statistics were used for analysis, which varied according to the distribution of the data. Comparisons of the frequency (number of transients per minute) of the dopamine concentration transients in different experimental conditions were made 


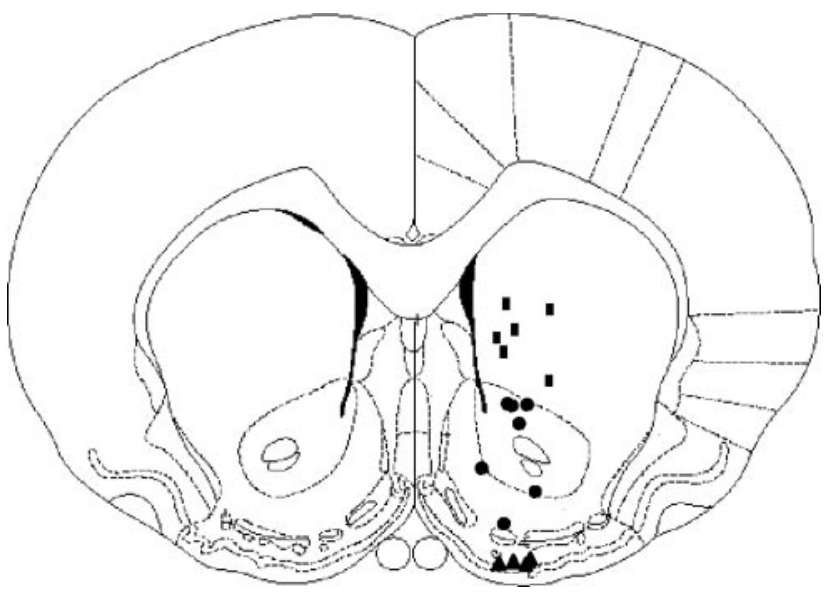

Figure 1. Representation of the carbon-fiber microelectrode recording sites in the dorsal and ventral striatum, as constructed from histological sections. The rectangles, circles, and triangles represent placements in the $\mathrm{CP}, \mathrm{NA}$, and OT, respectively.

with a Poisson distribution regression model with repeated measures. Comparisons of transient duration and $[\mathrm{DA}]_{\max }$ were made with a gamma distribution regression model with repeated measures. Comparisons of behaviors $5 \mathrm{sec}$ before and after dopamine transients were made with a binomial distribution regression model with repeated measures. Interactions, main effects, and pairwise contrasts were tested using Wald statistics. All calculations were performed using SAS software (SAS Institute, Inc., Cary, NC) on consultation with Statistical Services at the Odum Institute at the University of North Carolina.

\section{RESULTS}

\section{Histological verification of carbon-fiber placement}

The recording sites for the 17 male rats are illustrated in Figure 1. The dorsal and ventral striatum were divided into three broad regions: $\mathrm{CP}, \mathrm{NA}$, and $\mathrm{OT}$. Six recordings were made in the $\mathrm{CP}$ (rectangles), seven in the NA (circles) and four in the OT (triangles).

\section{Dopamine concentration transients during solitude and conspecific episodes}

A total of 125 dopamine concentration transients, identified by cyclic voltammograms, were detected during the first phase of the experiment, which evaluated dopamine transients during solitary and conspecific episodes in two consecutive presentation sets over a total of $26 \mathrm{~min}$. Transients occurred in both solitary and conspecific episodes in the 17 male rats tested (range, 1-22 transients per animal; mean, $7.4 \pm 1.5$; median, 5). Transients were observed in the CP, NA, and OT (vide infra).

The frequency of dopamine concentration transients was sixfold greater during episodes with conspecifics compared with solitary episodes (Fig. 2A). The Poisson distribution regression model yielded significant main effects of episode $\left(z_{1}=94.10 ; p<\right.$ $0.0001)$ as well as striatal region $\left(z_{2}=3.99 ; p<0.001\right)$. Pairwise comparisons demonstrated significant differences between the $\mathrm{CP}$ and the other nuclei (CP vs NA, $z_{1}=-3.75, p<0.001 ; \mathrm{CP}$ vs OT, $\left.z_{1}=-2.62, p<0.01\right)$. Thus, although dopamine transients were seen in each brain region tested, they were most common in the NA and OT.

The duration of the dopamine transients varied by experimental episode but not by brain region (Fig. 2B) (main effect of episode, $\left.z_{1}=3.87, p>0.0001\right)$. The duration of transients during conspecific episodes was $40 \%$ longer than during solitary episodes (conspecific episodes, $600 \pm 40 \mathrm{msec}$; range, 300-1600; solitary
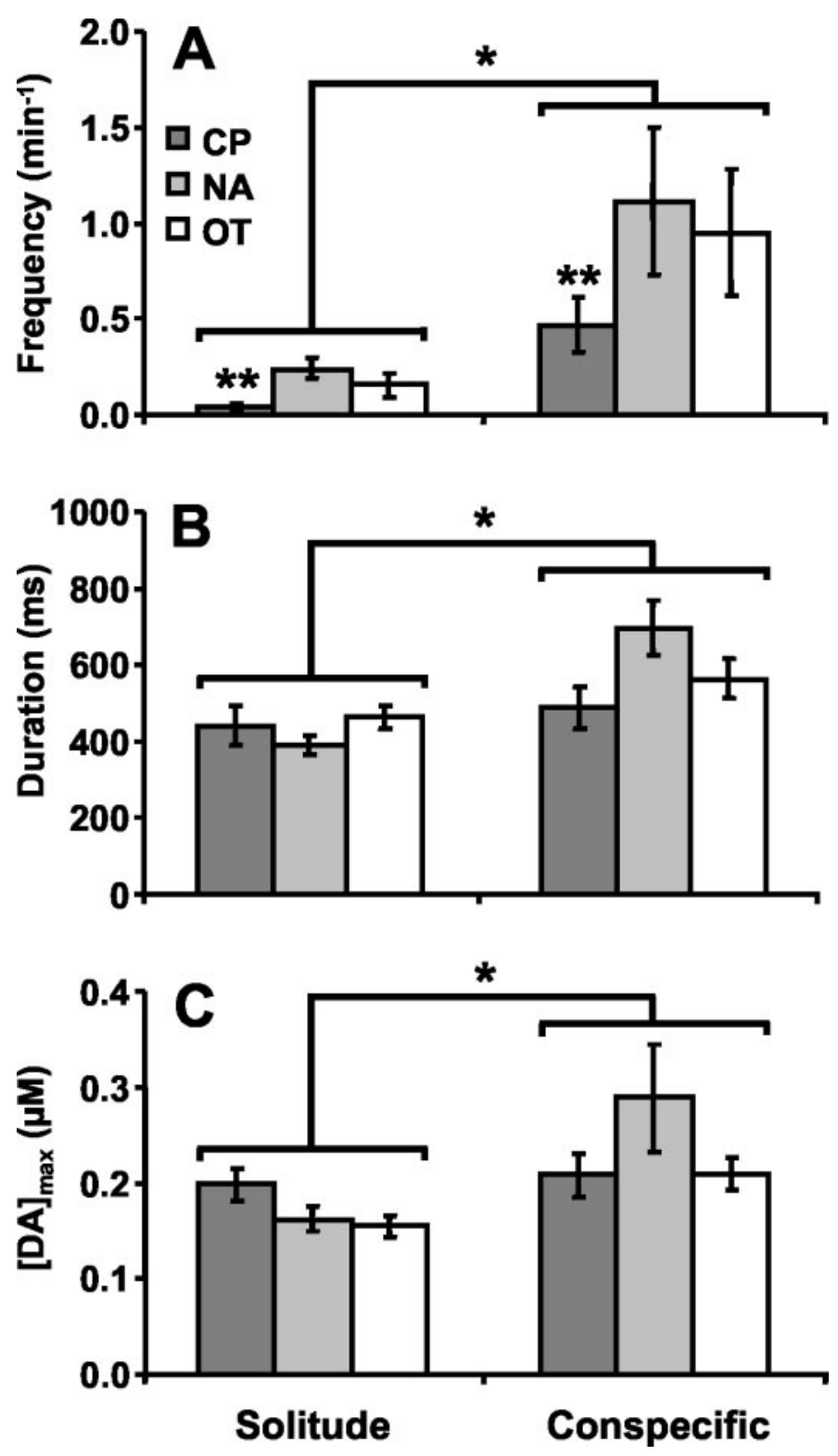

Figure 2. Dopamine concentration transients in the CP, NA, and OT: solitude versus brief conspecific interaction. $A$, Frequency of transients by brain region and episode; ${ }^{*} p<0.0001 ;{ }^{* *} p<0.01$, less frequent than NA and OT. $B$, Duration of transients by brain region and episode; * $p<$ 0.0001 . $C$, Peak concentration of transients by brain region and episode; ${ }^{*} p<0.01$.

episodes, $440 \pm 20 \mathrm{msec}$; range, 200-900). The [DA $]_{\max }$ (peak amplitude) had a similar profile (Fig. 2C), because transients observed during conspecific episodes were $50 \%$ larger than those observed during solitary episodes (main effect of episode, $z_{1}=$ $2.73, p>0.01)$. The mean $[\mathrm{DA}]_{\max }$ of the transients during brief conspecific episodes was $240 \pm 20 \mathrm{~nm}$ (range, 80-1350), whereas that of the transients during solitary episodes was $160 \pm 10 \mathrm{nM}$ (range, 60-330).

Dopamine transients were not exclusively associated with any one discrete event. However, $50 \%$ of the transients were observed for $5 \mathrm{sec}$ before and after the introduction of the conspecific to the test chamber; $25 \%$ of the transients occurred within $1 \mathrm{sec}$. The distribution of dopamine transients within the interaction episodes is presented in Figure 3, illustrating that phasic dopamine 


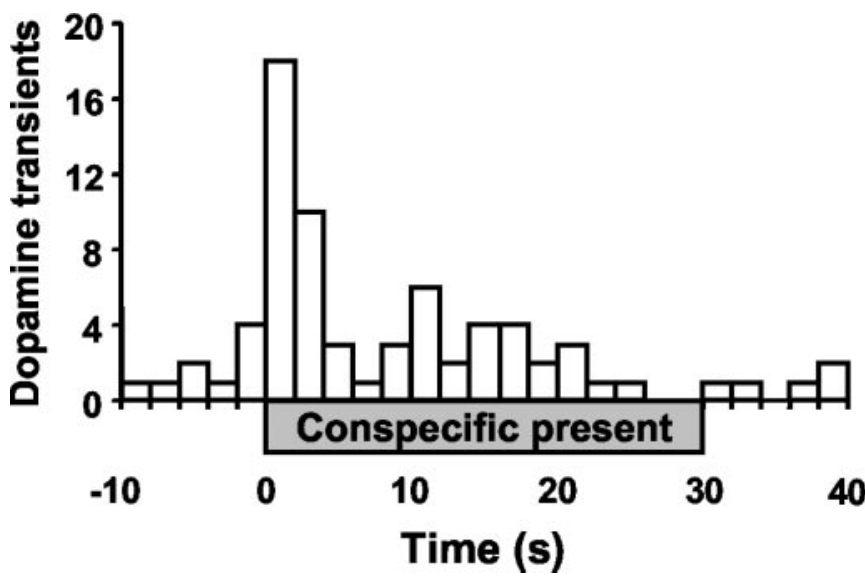

Figure 3. Histogram of dopamine concentration transients during the brief conspecific interaction episodes. Transients from all striatal regions and all interaction episodes were pooled into $2 \mathrm{sec}$ bins, with the introduction of the conspecific at time 0 . The presence of the conspecific is denoted by the gray bar from 0 to $30 \mathrm{sec}$.

activity clustered around the introduction of the conspecific to the test chamber. This effect was not simply a consequence of a foreign object entering the test chamber, because there was no cluster of transients around the removal of the conspecific, which required insertion of the experimenter's hand into the cage.

Examples of dopamine concentration transients recorded at the start of a brief conspecific episode are shown in Figure 4. A sequence during the presentation of a receptive female is shown in Figure $4 C$, whereas the trace in Figure $4 A$ shows the changes in dopamine concentration in the OT of the male recorded by fast-scan cyclic voltammetry over the same time span (4 sec). The numbers in the top right corner of the video stills indicate the corresponding cyclic voltammogram, imprinted on the video record by the video character generator. Before presentation, the male was sitting quietly on an absorbent pad in the chamber and the concentration changes stayed within the noise level (Fig. $4 C$, left ). Although basal concentrations cannot be measured with this technique, transient concentration changes are readily discerned, as seen in the middle of the figure. As the female entered the test chamber (Fig. 4C, middle), a transient dopamine concentration change occurred; because the male faced away from the female, the sensory trigger was presumably auditory. Immediately after the transient, the male oriented toward the conspecific. A second transient occurred as the male made whisker contact with the female (Fig. 4C, right). The whisker contact was easily discernible in an enlarged video image (data not shown). Dopamine was identified by its cyclic voltammogram (Fig. $4 B$ ); those recorded at the maximum concentration changes have the features of a dopamine cyclic voltammogram. This is shown by the overlap of the cyclic voltammograms with one recorded in this animal during the electrical stimulation of the dopaminergic pathway (dotted line, scaled to size). The durations of transients in this example are $0.8 \mathrm{sec}$ (middle) and $0.4 \mathrm{sec}$ (right); the electrochemical scans confirmed to be dopamine are shown by open circles. This sequence was followed by the male initiating sniffing of the female.

\section{Habituation of dopamine concentration transients during conspecific episodes}

The frequency of dopamine transients within conspecific episodes was found to decrease during the second presentation set, indicating habituation of dopamine transients (Fig. 5A). Although there was no significant interaction between the brain region and the presentation set, the main effect of the set was significant

Figure 4. Two dopamine concentration transients in the OT of a male rat associated with the introduction of a receptive female to the test chamber and subsequent contact. $A, \mathrm{~A} 4 \mathrm{sec}$ trace of the electrochemical signal at the oxidation potential of dopamine $(+0.6 \mathrm{~V}$ vs $\mathrm{Ag} / \mathrm{AgCl}$ reference) converted to dopamine concentration using the in vitro calibration of the electrode after the experiment. The electrochemical scans confirmed to be dopamine by the cyclic voltammograms are indicated by open circles. $B$, Cyclic voltammograms corresponding to the electrochemical signal at the times indicated by the arrows. The cyclic voltammograms verify that the changes in electrochemical signal during the two transients are attributable to the oxidation of dopamine (middle and right), whereas no changes in dopamine are apparent beforehand (left). The oxidative and reductive peaks of the dopamine transients are compared with those of dopamine obtained during the electrical stimulation of the dopamine fibers (dotted line, scaled to size). $C$, The video record of the experiment corresponding to the electrochemical signal at the times indicated by the arrows. The first dopamine transient coincided with the entrance of

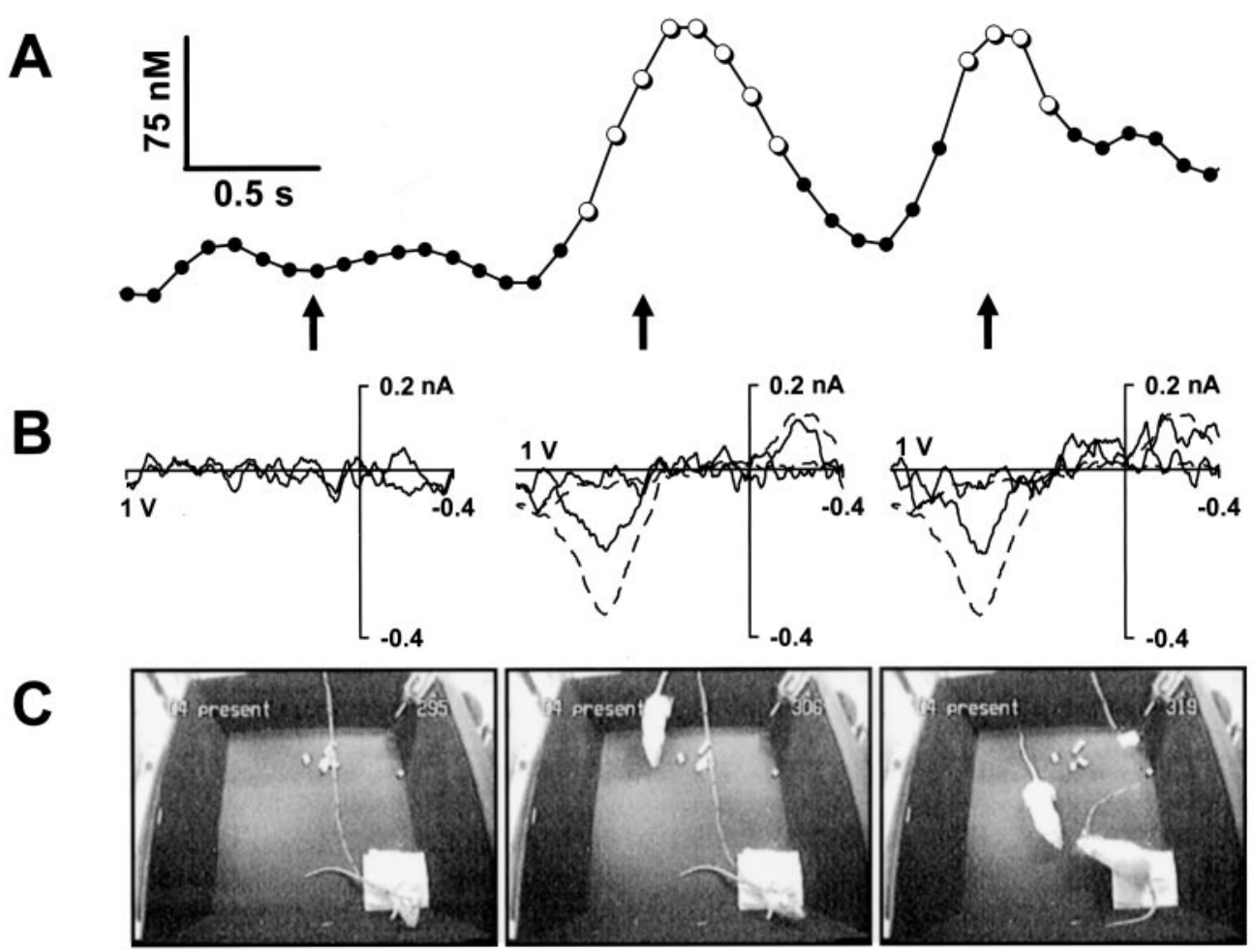
the female to the test chamber and was followed by immediate orientation of the male toward the female. The second transient was concurrent with the initial whisker contact between the rats and was followed by investigative sniffing of the female by the male. 

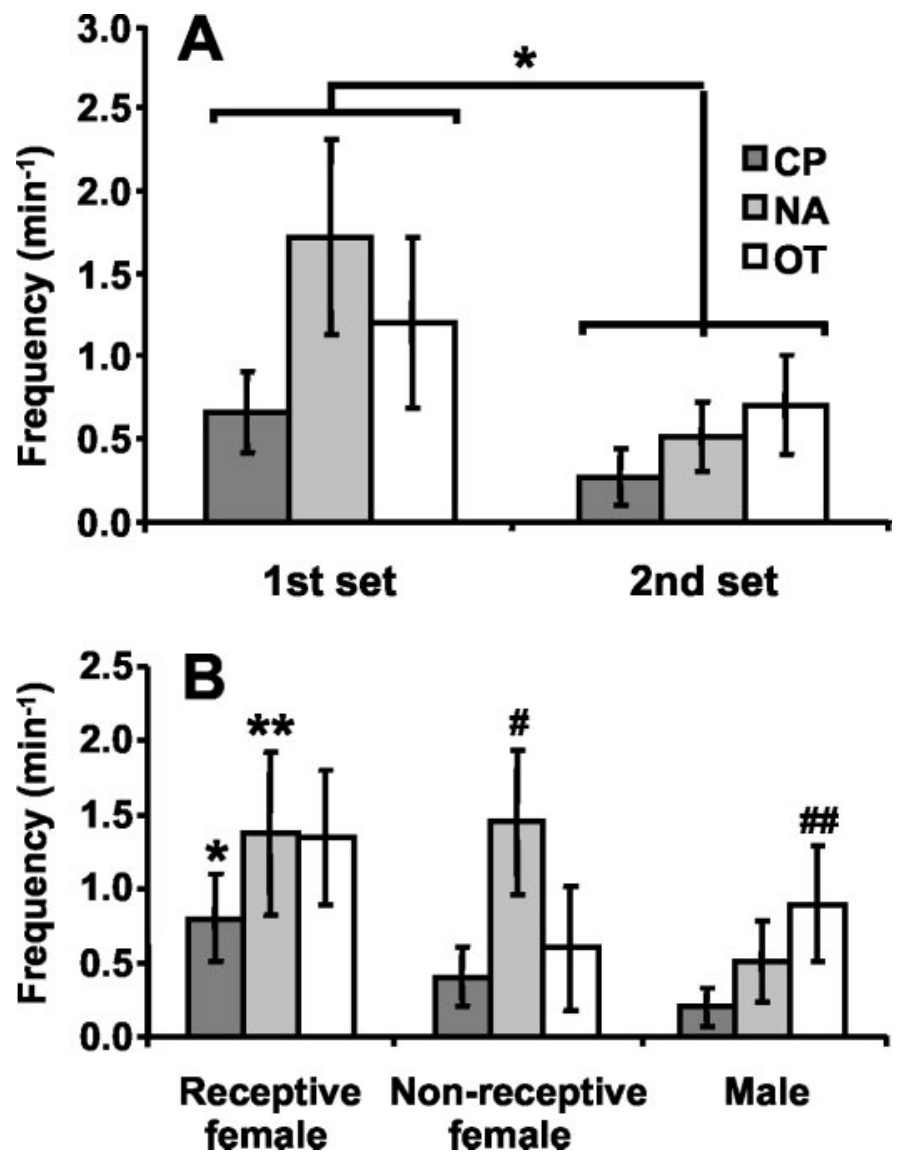

Figure 5. Frequency of dopamine concentration transients during brief conspecific episodes: effects of presentation set and conspecific rat. $A$, Habituation of transients during the second presentation set, by brain region; ${ }^{*} p<0.0001$. $B$, Frequency of transients in the presence of different conspecific rats, by brain region; ${ }^{*} p<0.05$, greater than $C P$ nonreceptive female; ${ }^{* *} p<0.05$, greater than NA male; ${ }^{*} p<0.05$, greater than NA male, greater than $\mathrm{CP}$ nonreceptive female; ${ }^{\# \#} p<0.05$, greater than $\mathrm{CP}$ male.

$\left(z_{1}=4.01 ; p<0.0001\right)$, indicating that the frequency of transients decreased by $60 \%$ during the second presentation set. In contrast, significant habituation did not occur within the first presentation set. The frequencies of dopamine transients (per minute, pooled across striatal regions) during the individual conspecific episodes were: first, $1.1 \pm 0.3$; second, $1.4 \pm 0.3$; third, $1.1 \pm 0.3$; fourth, $0.6 \pm 0.1$; fifth, $0.4 \pm 0.1$; and sixth, $0.4 \pm 0.1$. Only the fifth and sixth presentations were significantly different from the first (first vs fifth, $z_{1}=3.17, p<0.01$; first vs sixth, $\left.z_{1}=2.16, p<0.05\right)$. Moreover, the habituation was not attributable to an altered response of the carbon-fiber electrode or to an inability of the surrounding tissue to support transients, because the amplitude of electrically evoked dopamine signals did not change across the experiment (paired $t$ test; $p>0.05$; data not shown). Anecdotally, we note that the habituation was not attributable to a general decrease in activity, because the rats appeared to be more responsive to the conspecific and more behaviorally invigorated during the second presentation set.

\section{Dopamine concentration transients during episodes with females compared with males}

Across all striatal regions, dopamine transients were 2.4 times more frequent when receptive females were present and 1.8 times more frequent when nonreceptive females were present compared with males (Fig. 5B). The Poisson distribution regression model yielded a significant interaction between the brain region and the type of conspecific rat $\left(z_{4}=3.16 ; p<0.05\right)$. Subsequent pairwise comparisons showed that in the $\mathrm{CP}$, the frequency of dopamine signals with a receptive female present were marginally higher than with a male $\left(z_{1}=1.90 ; p<0.058\right)$ and significantly higher than with a nonreceptive female $\left(z_{1}=2.26\right.$; $\left.p<0.05\right)$. In the NA, dopamine transients were observed as frequently with receptive as with nonreceptive females, but significantly less often with males (males vs receptive females, $z_{1}=-2.38, p<0.05$; males vs nonreceptive females, $\left.z_{1}=-2.35, p<0.05\right)$. In the OT, there was a trend for increased transients during the presence of receptive females that did not reach statistical significance (receptive females vs males, $z_{1}=1.72, p<0.09$ ). Among brain regions, the frequency of the transients during the presence of nonreceptive females was significantly higher in the NA than in the CP $\left(z_{1}=2.34 ; p<0.05\right)$. Finally, transients associated with the presence of males were significantly higher in the OT than in the $\mathrm{CP}\left(z_{1}=2.19 ; p<0.05\right)$.

\section{Association of dopamine concentration transients with specific behaviors during conspecific episodes}

The behavior of the test rat before and after each dopamine concentration transient during conspecific episodes was evaluated in $1 \mathrm{sec}$ increments. The compiled behavior for all transients observed during the conspecific episodes is shown in Figure 6 for each brain region. In the left panels, the $y$-axis designates the counts of particular behaviors, with the maximum value as the number of dopamine transients observed in the respective striatal region; thus, it is readily apparent that no one behavior was associated with every transient. Nevertheless, increases in behavioral activation can be seen after the dopamine transients (Fig. 6, left), with the most robust increase being in sniffing the conspecific. For statistical analysis, the sum of the scores for each behavior in the $5 \mathrm{sec}$ interval after the dopamine transient was compared with the sum of the scores for the $5 \mathrm{sec}$ before (Fig. 6 , right). With $70 \%$ of the transients in the CP, $50 \%$ of those in the $\mathrm{NA}$, and $40 \%$ of those in the OT, sniffing of the conspecific increased after the transient $\left(\mathrm{CP}, z_{1}=2.28, p<0.05\right.$; NA, $z_{1}=$ 2.42, $p<0.05$; OT, $\left.z_{1}=1.94, p<0.053\right)$. In the NA, other behaviors were also increased after the dopamine transient. General sniffing increased in $60 \%$ of the transients $\left(z_{1}=2.51 ; p<\right.$ $0.05)$, orienting behaviors increased in $60 \%\left(z_{1}=3.38 ; p<0.001\right)$, and locomotion to the rat increased in $30 \%\left(z_{1}=2.59 ; p<0.01\right)$. There was no association between dopamine concentration transients and changes in sexual behavior, $22 \mathrm{kHz}$ ultrasonic vocalizations, or grooming (data not shown).

Similar results were seen when the data were pooled across brain regions and separated according to the type of conspecific rat presented (data not shown). In that analysis, general sniffing associated with dopamine transients did not significantly change, whereas both orienting behaviors and sniffing the conspecific increased with similar magnitude in all groups. The increase in orientation reached significance only in the presence of nonreceptive females $\left(z_{1}=2.28 ; p<0.05\right)$; the increase in sniffing the conspecific reached significance in the presence of both females (receptive females, $z_{1}=3.19, p<0.01$; nonreceptive females, $\left.z_{1}=3.40, p<0.001\right)$. In contrast, locomotion to the rat increased in the presence of both female conspecifics and was statistically significant in the presence of nonreceptive females $\left(z_{1}=2.85 ; p<\right.$ 0.01 ) but was minimal and did not change in the presence of males. 

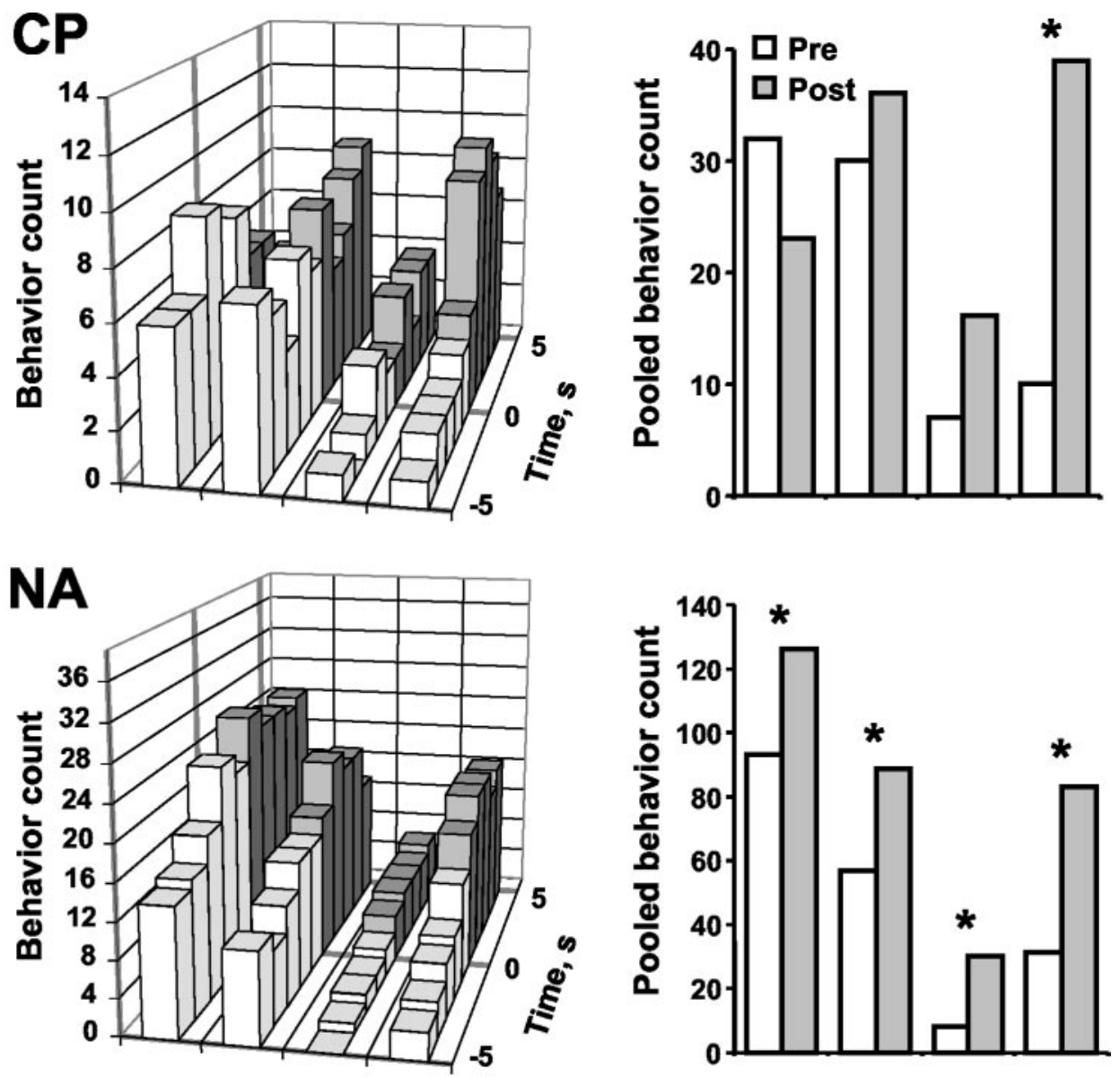

Figure 6. Behaviors before and after dopamine concentration transients during conspecific episodes. Left, Behaviors were scored for $5 \mathrm{sec}$ before (white bars) and 5 sec after (gray bars) the start of each dopamine transient; the transient occurred at time 0 . The $y$-axis designates the counts of particular behaviors, with the maximum value considered to be the number of dopamine transients observed in the respective brain region. The $x$-axis shows seconds before and after the dopamine transient; the $z$-axis displays various behaviors. Right, The behavior counts from the associated left panels were pooled into before (Pre; white bars) and after (Post; gray bars) totals. Dopamine transients in the $\mathrm{CP}$ were significantly associated with increased sniffing of the conspecific. Transients in the NA were significantly associated with increases in general sniffing and orienting movements, as well as approach to and sniffing the conspecific; ${ }^{*} p<0.05$, before versus after.
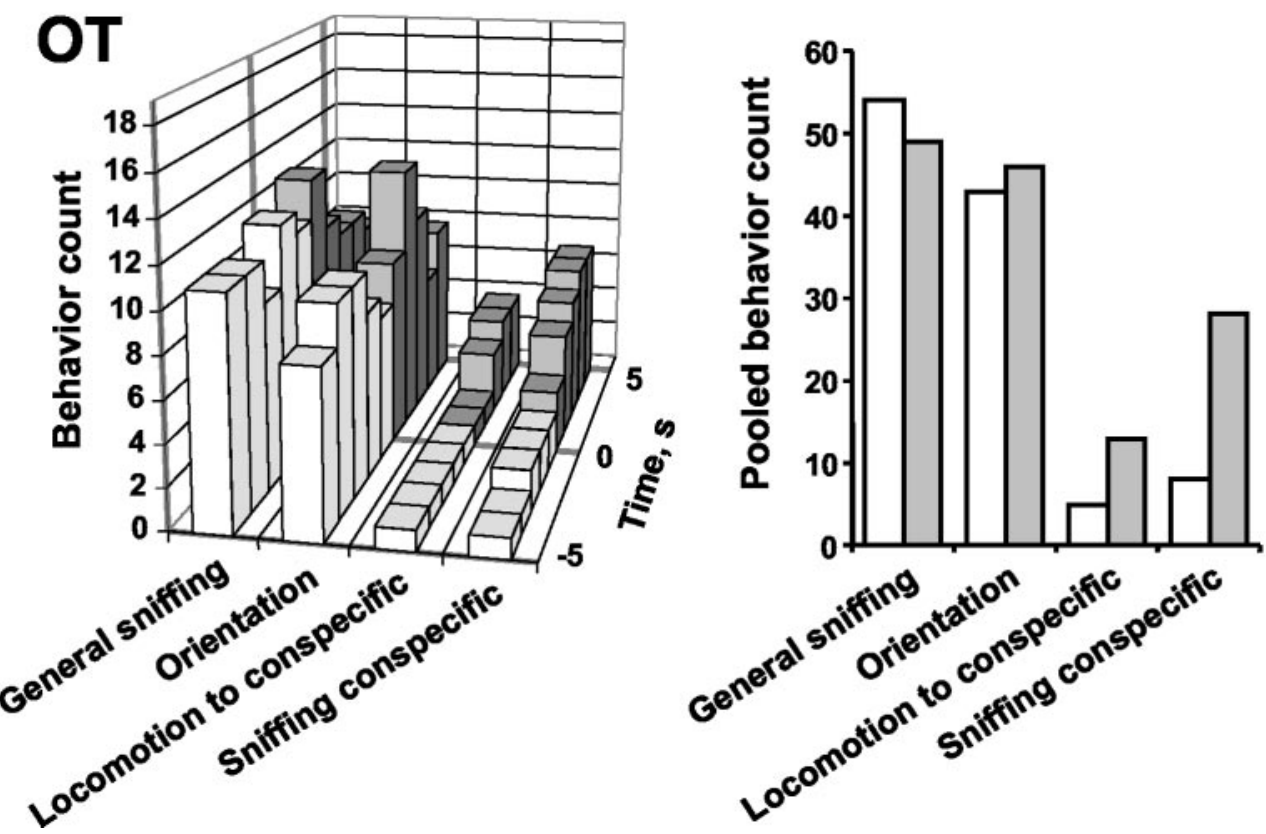

\section{Copulatory behavior}

Seven of the males performed at least one intromission and one male ejaculated during the brief conspecific episodes. During the copulation phase of the experiment, 12 of the 17 male rats copulated (six rats from the CP group, three rats from the NA group, and three rats from the OT group). The rats that engaged in copulation during the copulation phase exhibited $5 \pm 1$ simple mounts with an $8 \pm 2$ sec latency to mount and $7 \pm 1$ intromissions with a $56 \pm 37 \mathrm{sec}$ latency to intromit and a $20 \pm 2 \mathrm{sec}$ inter-intromission interval. The ejaculation latency was $197 \pm 80$ sec, and the subsequent vocalization latency was $216 \pm 94 \mathrm{sec}$. Thus, males that copulated did so quite rapidly; however, the fact 

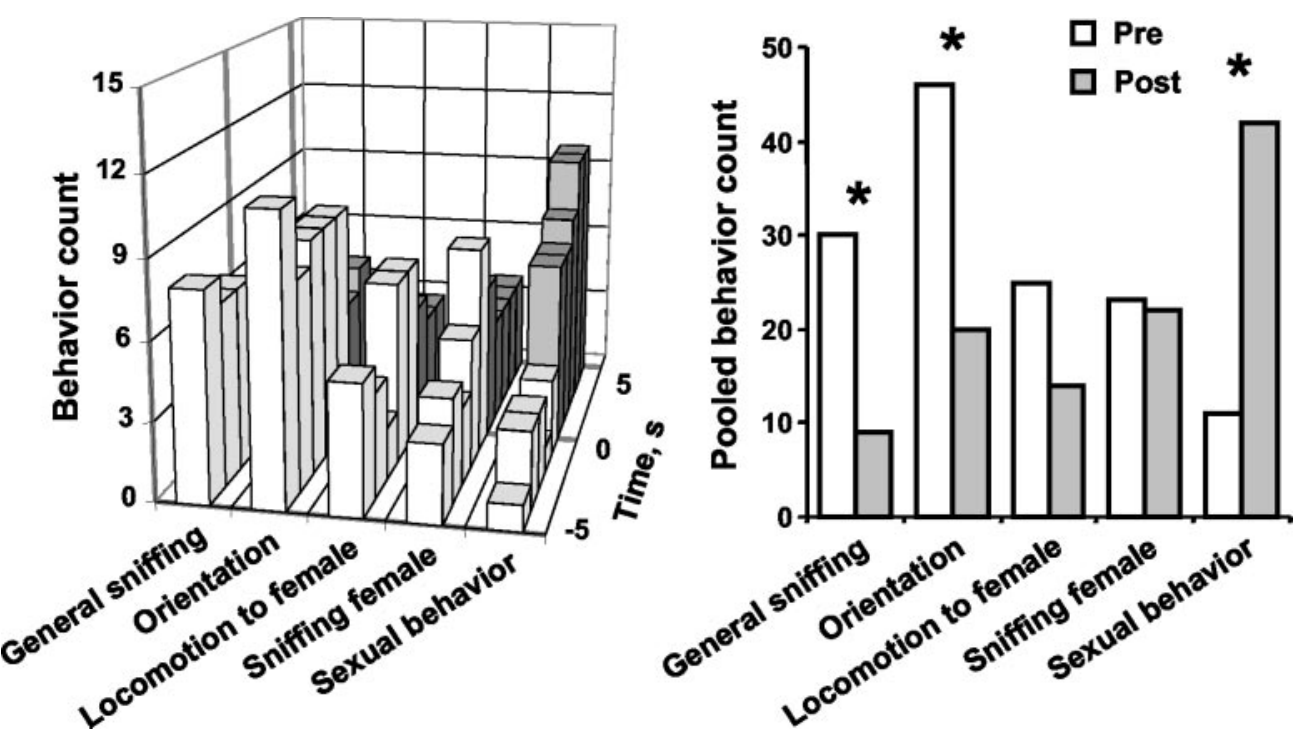

Figure 7. Behaviors before and after dopamine concentration transients during copulation episodes. Left, Behaviors were scored for $5 \mathrm{sec}$ before (white bars) and $5 \mathrm{sec}$ after (gray bars) the start of each dopamine transient; the transient occurred at time 0 . The $y$-axis designates the counts of particular behaviors, with the maximum value considered to be the number of dopamine transients observed. The $x$-axis shows seconds before and after the dopamine transient, whereas the $z$-axis displays various behaviors. Right, The behavior counts from the associated left panels were pooled into before (Pre; white bars) and after (Post; gray bars) totals. Dopamine transients were significantly associated with increases in sexual behaviors and with decreases in general sniffing and orienting behaviors; ${ }^{*} p<0.05$, before versus after. that the receptive female was available only briefly before the copulation episode likely influenced the speed of subsequent copulation (Sachs and Barfield, 1976).

\section{Dopamine concentration transients during copulation episodes}

A total of 15 dopamine concentration transients were detected in the 12 rats that copulated. The number of transients observed in each rat ranged from 0 to 3 , with a mean of $1.3 \pm 0.3$ and a median of 1 . The number of rats that copulated and the frequency of the transients in those rats were too small to compare frequencies among brain regions. The overall frequency of dopamine transients during copulation was $0.17 \pm 0.05$ per minute, which is $20 \%$ of that during the brief conspecific episodes and $120 \%$ of that during the solitary episodes. The $[\mathrm{DA}]_{\max }$ was $190 \pm 20 \mathrm{nM}$ (range, 90-330), and the average duration was $390 \pm 30 \mathrm{msec}$ (range, 300-600). Interestingly, 26\% of the transients occurred 5 sec before and after the introduction of the receptive female.

The compiled behavior of the test rats $5 \mathrm{sec}$ before and after each dopamine transient during the copulation episode is shown in Figure 7 . With $50 \%$ of the transients, general sniffing decreased $\left(z_{1}=-2.2 ; p<0.05\right)$, and with $60 \%$ of the transients, orienting movements decreased $\left(z_{1}=-2.81 ; p<0.01\right)$. Moreover, twothirds of the dopamine transients were followed by increases in sexual behavior $\left(z_{1}=3.78 ; p<0.001\right)$. There were no significant associations with changes in grooming (data not shown), sniffing the female, or locomotion.

\section{Dopamine concentration transients associated with intromission}

Eleven dopamine transients (five in $\mathrm{CP}$, four in NA, and two in OT) occurred in the $5 \mathrm{sec}$ leading to intromission; two of these transients were observed during the brief conspecific episodes, whereas the remaining transients were observed during the copulation period. Five of these transients were observed in the 2-5 sec before the intromission, whereas the remaining six occurred within $1 \mathrm{sec}$. A histogram of the transients along the intromission sequence is shown in Figure 8. "Mount" was defined as the time from when the forepaws were placed on the female's back to the beginning of thrusting and lasted $200-400 \mathrm{msec}$; thrusting lasted 200-600 msec. "Dismount" was defined as the time from when the male's forelimbs splayed to the conclusion of the jump away

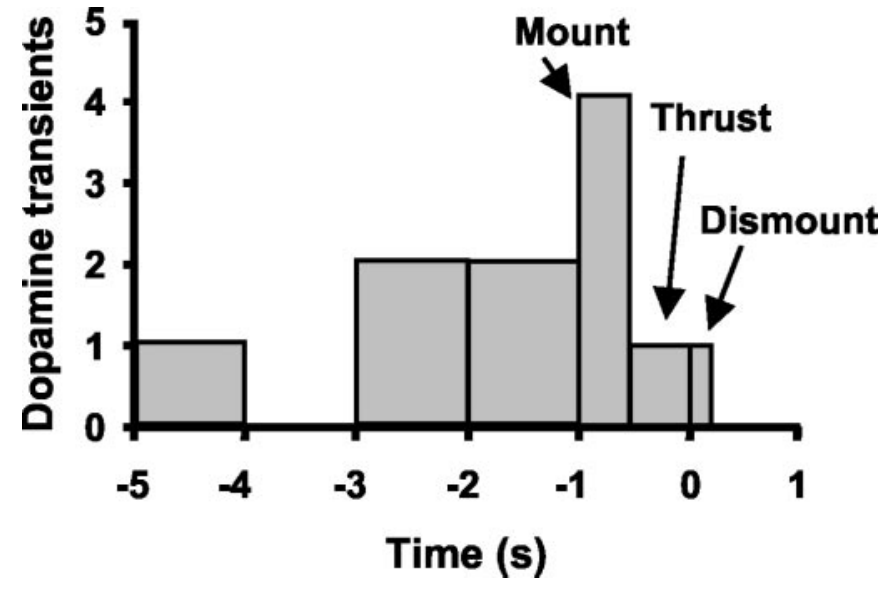

Figure 8. Histogram of dopamine concentration transients associated with intromissions. The $x$-axis displays time, with the end of intromission at $0 \mathrm{sec}$. See Results for operational definitions of mount, thrust, and dismount. The majority of transients occurred before the consummatory aspect of intromission (i.e., thrusting).

from the female and lasted 100-200 msec. Strikingly, $82 \%$ of these transients occurred before what would be considered the consummatory phase of intromission (i.e., before thrusting and penetration of the penis).

\section{DISCUSSION}

These studies reveal that dopamine concentration transients naturally occur in the CP, NA, and OT of male rats. The frequency of the transients was low during solitude and copulation and increased dramatically during brief conspecific interaction, particularly at the introduction of the conspecific to the test chamber. Although dopamine transients were more frequent in the ventral striatum, the overall characteristics of the signals were remarkably similar among striatal regions. They reached concentrations similar to the $K_{\mathrm{m}}$ for the dopamine transporter ( $\left.200 \mathrm{nM}\right)$ (Near et al., 1988), sufficient to interact with some low-affinity receptors (Richfield et al., 1989), and lasted for only $\sim 0.5 \mathrm{sec}$. The frequency of dopamine transients was highest at the initial presentation of females. The phasic dopamine response to a conspecific habituated with a second encounter. During copulation, dopa- 
mine concentration transients continued, although their frequency was much lower than during the initial conspecific interactions. Two-thirds of the transients observed during copulation were related to sexual behaviors, and the majority of these preceded consummatory aspects. These results establish for the first time that transient dopamine concentrations occur throughout the dorsal and ventral striatum, and show that they are more frequent with biologically salient stimuli.

\section{Dopamine concentration transients}

These electrochemical recordings provide a unique view of brain dopaminergic activity, revealing dopamine fluctuations on a time scale similar to burst firing. In contrast to microdialysis, which integrates samples collected over minutes from millimeters of tissue, the carbon-fiber electrode provides subsecond measurements of dopamine from discrete anatomical locations. Moreover, unlike other electrochemical reports (for review, see Wightman and Robinson, 2002), the signal has been established as endogenous dopamine by multiple criteria. First, dopamine transients were identified by computer-matching of the voltammograms and verified subsequently by human inspection. Second, recordings were made in anatomical sites selected because they supported robust dopamine release during electrical stimulation of the ascending dopaminergic processes. Third, we demonstrated previously that the duration and the number of transients increase after pharmacological inhibition of dopamine uptake (Robinson et al., 2001). Thus, unlike previous reports of "dopamine-like signals," the transients shown here are attributable to dopamine released into the extracellular space.

A fourth indication that dopamine is the identified species is the concurrence with electrophysiological data. Dopaminergic neurons periodically burst fire, and these bursts have been hypothesized to generate dopamine transients (Schultz, 1998). Burst firing of dopaminergic neurons is found in nonhuman primates, cats, and rats in response to a variety of stimuli. These include the unexpected presentation of rewards (Romo and Schultz, 1990; Mirenowicz and Schultz, 1996), conditioned stimuli (Mirenowicz and Schultz, 1994), discriminative stimuli (Miller et al., 1981), and novelty (Steinfels et al., 1983; Freeman and Bunney, 1987; Ljungberg et al., 1992). The bursts occur with the synchrony of many neurons (Grace, 1991), similar to the synchrony induced during electrical stimulation. Indeed, the naturally occurring concentration transients reported here closely resemble dopamine release during high-frequency stimulation of dopaminergic neurons (Robinson et al., 2001). The higher frequencies of transients in ventral versus dorsal striatum may reflect differences in bursting rates between the ventral tegmental area and the substantia nigra (Overton and Clark, 1997).

For neurons recruited to fire together, the $[\mathrm{DA}]_{\max }$ depends on the release rate and burst frequency, whereas its extracellular lifetime will depend on uptake (Wightman and Zimmerman, 1990). It is known that release and uptake rates are regionally specific, with larger rates in the CP (Garris and Wightman, 1994; Jones et al., 1995). Thus, we were surprised at the lack of variation in the amplitude and duration of the measured signals among regions. Instead, release and uptake rates balanced, leading to similar transients in the different nuclei. In contrast, Rebec et al. (1997) reported dopamine transients associated with novelty in the NA shell but not the core. As fast voltammetric measurements are made in a variety of situations, the nature of phasic dopamine activity in the striatum will be revealed.

\section{Association of dopamine concentration transients with behavior}

Although stimuli that promote burst firing of dopaminergic neurons often provoke behavioral responses, the bursting is not correlated with specific motor movements (Nishino et al., 1987; Romo and Schultz, 1990). Similarly, the dopamine transients reported here do not correlate with any one behavior. The signals observed during solitude, which are presumably distinct from environmental cues, may arise from bursts similar to those observed in anesthetized animals (Grace and Bunney, 1980). Like burst firing (Overton and Clark, 1997), the dopamine transients observed in the presence of conspecifics were apparently triggered by stimuli communicated through various sensory modalities. For example, dopamine transients that occurred during the introduction of a conspecific could arise from visual or auditory input. Moreover, some dopamine transients were associated with sniffing the conspecific, providing olfactory or tactile input. After the dopamine signals, the rat displayed various appetitive behaviors toward the conspecific. These variable causes and responses are as expected for a circuit in the basal ganglia network that underlies flexible voluntary movements (Pennartz et al., 1994).

The open-field experimental design permitted an assortment of potential stimuli. We purposely used this design because the occurrence of dopamine transients was previously uncharacterized. The findings suggest that a variety of stimuli are effective, with the highest incidence during introduction of a conspecific. On the second presentation set, the signal habituated, suggesting that novelty or expectation contributed to the incidence of dopamine transients. Notably, habituation to the repeated presentation of a novel stimulus has been reported for single-unit phasic activity (Ljungberg et al., 1992) and for dopamine transients (Rebec et al., 1997). Habituation may also contribute to the lower frequency of dopamine signals during copulation. However, the number of transients was fewer in the presence of males compared with females, suggesting that a difference in biological salience also contributes to these signals.

\section{Extracellular dopamine: phasic differs from tonic}

On the time scale of minutes, dopamine concentrations increase during sexual behavior, as measured with microdialysis (Pfaus et al., 1990; Pleim et al., 1990; Fiorino et al., 1997; Lorrain et al., 1999). However, based on quantitative microdialysis calibrations (Justice, 1993), the concentration changes observed in those experiments were $<100 \mathrm{~nm}$ and existed for a prolonged period (tens of minutes). These changes may reflect a variation in the lowfrequency tonic firing of dopaminergic neurons or glutamatergic actions at dopaminergic terminals (Grace, 2000). Because dopamine transients are difficult to measure with microdialysis (Lu et al., 1998), phasic dopamine changes are unlikely to contribute to the dialysis signal. Even previous electrochemical recordings of dopamine during sexual behavior (Mas et al., 1990; Mitchell and Gratton, 1991) were on a minute time scale, obscuring transient changes. Unfortunately, fast-scan cyclic voltammetry cannot monitor slow changes in dopamine, and thus cannot address tonic changes. However, the advantage of the time resolution in the present experiments is highlighted in Figure 8, because dopamine transients can be matched to specific phases of the intromission event.

Tonic and phasic dopamine neurotransmission, each causing different changes in dopamine extracellular concentrations, may play distinct functional roles that influence behavior. The role of dopamine, especially in reward, has received extensive theoretical 
consideration (Wise and Rompre, 1989; Salamone et al., 1997; Berridge and Robinson, 1998; Ikemoto and Panksepp, 1999; Berke and Hyman, 2000). However, most pertinent to our observations are theories addressing phasic aspects of dopaminergic function (Schultz, 1998; Redgrave et al., 1999). Schultz (1998) proposed that phasic firing of dopaminergic neurons predicts reward in reinforcement learning from studies on firing during associative learning in primates. Our data were collected within a nonlearning paradigm and do not address this theory directly. Nevertheless, they are consistent in that dopamine transients occur with the unexpected presentation of a conspecific. Furthermore, the habituation of the dopamine response could be attributable to increased expectation of another conspecific presentation. Redgrave et al. (1999) offered a more general explanation: phasic dopamine contributes to switching attention to a salient stimulus. In this role, phasic dopamine participates not only in associative learning but more broadly in response facilitation (Oades, 1985; Salamone et al., 1997; Ikemoto and Panksepp, 1999). Our data are also consistent with this theory, because dopamine transients were often followed by an increase in behaviors toward the conspecific. Nevertheless, until simultaneous electrophysiological and electrochemical measurements are made from dopamine neurons, the precise relationship between dopamine burst firing and concentration transients will remain unknown.

\section{Conclusion}

The present results characterize dopamine concentration transients in multiple brain regions of male rats during biologically relevant situations. Current neurochemical data support the electrophysiological evidence of two parallel modes of dopamine signaling (Wightman and Robinson, 2002). Tonic dopamine results in low concentrations that are readily sampled by microdialysis and convey information through high-affinity receptors (Richfield et al., 1989). Phasic dopamine results in concentration transients that are best sampled by rapid electrochemical techniques. These higher concentrations saturate the transporter, allowing dopamine to participate in long-range volume transmission (Garris et al., 1994) and cause different interactions with receptors (Williams and Millar, 1990; Gonon, 1997). The present results are consistent with current theories of phasic dopamine function, but the precise causes and consequences of the extrasynaptic signals require additional exploration.

\section{REFERENCES}

Berke JD, Hyman SE (2000) Addiction, dopamine, and the molecular mechanisms of memory. Neuron 25:515-532.

Berridge KC, Robinson TE (1998) What is the role of dopamine in reward: hedonic impact, reward learning, or incentive salience? Brain Res Brain Res Rev 28:309-369.

Fiorino DF, Coury A, Phillips AG (1997) Dynamic changes in nucleus accumbens dopamine efflux during the Coolidge effect in male rats. J Neurosci 17:4849-4855.

Freeman AS, Bunney BS (1987) Activity of A9 and A10 dopaminergic neurons in unrestrained rats: further characterization and effects of apomorphine and cholecystokinin. Brain Res 405:46-55.

Garris PA, Wightman RM (1994) Different kinetics govern dopaminergic transmission in the amygdala, prefrontal cortex, and striatum: an in vivo voltammetric study. J Neurosci 14:442-450.

Garris PA, Ciolkowski EL, Pastore P, Wightman RM (1994) Efflux of dopamine from the synaptic cleft in the nucleus accumbens of the rat brain. J Neurosci 14:6084-6093.

Garris PA, Christensen JRC, Rebec GV, Wightman RM (1997) Realtime measurement of electrically evoked extracellular dopamine in the striatum of freely moving rats. J Neurochem 68:152-161.

Gonon F (1997) Prolonged and extrasynaptic excitatory action of dopamine mediated by $\mathrm{D} 1$ receptors in the rat striatum in vivo. J Neurosci 17:5972-5978.
Grace AA (1991) Phasic versus tonic dopamine release and the modulation of dopamine system responsivity: a hypothesis for the etiology of schizophrenia. Neuroscience 41:1-24.

Grace AA (2000) Gating of information flow within the limbic system and the pathophysiology of schizophrenia. Brain Res Brain Res Rev $31: 330-341$.

Grace AA, Bunney BS (1980) Nigral dopamine neurons: intracellular recording and identification with L-dopa injection and histofluorescence. Science 210:654-656.

Ikemoto S, Panksepp J (1999) The role of nucleus accumbens dopamine in motivated behavior: a unifying interpretation with special reference to reward-seeking. Brain Res Brain Res Rev 31:6-41.

Jones SR, Garris PA, Kilts CD, Wightman RM (1995) Comparison of dopamine uptake in the basolateral amygdaloid nucleus, caudateputamen, and nucleus accumbens of the rat. J Neurochem 64:2581-2589.

Justice Jr JB (1993) Quantitative microdialysis of neurotransmitters. J Neurosci Methods 48:263-276.

Kawagoe KT, Garris PA, Wiedemann DJ, Wightman RM (1992) Regulation of transient dopamine concentration gradients in the microenvironment surrounding nerve terminals in the rat striatum. Neuroscience 51:55-64.

Ljungberg T, Apicella P, Schultz W (1992) Responses of monkey dopamine neurons during learning of behavioral reactions. J Neurophysiol 67:145-163.

Lorrain DS, Riolo JV, Matuszewich L, Hull EM (1999) Lateral hypothalamic serotonin inhibits nucleus accumbens dopamine: implications for sexual satiety. J Neurosci 19:7648-7652.

Lu Y, Peters JL, Michael AC (1998) Direct comparison of the response of voltammetry and microdialysis to electrically evoked release of striatal dopamine. J Neurochem 70:584-593.

Mas M, Gonzalez-Mora JL, Louilot A, Sole C, Guadalupe T (1990) Increased dopamine release in the nucleus accumbens of copulating male rats as evidenced by in vivo voltammetry. Neurosci Lett 110:303-308.

Michael DJ, Joseph JD, Kilpatrick MR, Travis ER, Wightman RM (1999) Improving data acquisition for fast-scan cyclic voltammetry. Anal Chem 71:3941-3947.

Miller JD, Sanghera MK, German DC (1981) Mesencephalic dopaminergic unit activity in the behaviorally conditioned rat. Life Sci 29:1255-1263.

Mirenowicz J, Schultz W (1994) Importance of unpredictability for reward responses in primate dopamine neurons. J Neurophysiol 72:1024-1027.

Mirenowicz J, Schultz W (1996) Preferential activation of midbrain dopamine neurons by appetitive rather than aversive stimuli. Nature 379:449-451.

Mitchell JB, Gratton A (1991) Opioid modulation and sensitization of dopamine release elicited by sexually relevant stimuli: a high speed chronoamperometric study in freely behaving rats. Brain Res $551: 20-27$.

Near JA, Bigelow JC, Wightman RM (1988) Comparison of uptake of dopamine in rat striatal chopped tissue and synaptosomes. J Pharmacol Exp Ther 245:921-927.

Nishino H, Ono T, Muramoto K, Fukuda M, Sasaki K (1987) Neuronal activity in the ventral tegmental area (VTA) during motivated bar press feeding in the monkey. Brain Res 413:302-313.

Oades RD (1985) The role of noradrenaline in tuning and dopamine in switching between signals in the CNS. Neurosci Biobehav Rev 9:261-282.

Overton PG, Clark D (1997) Burst firing in midbrain dopaminergic neurons. Brain Res Brain Res Rev 25:312-334.

Pennartz CM, Groenewegen HJ, Lopes da Silva FH (1994) The nucleus accumbens as a complex of functionally distinct neuronal ensembles: an integration of behavioural, electrophysiological, and anatomical data. Prog Neurobiol 42:719-761.

Pfaus JG, Damsma G, Nomikos GG, Wenkstern DG, Blaha CD, Phillips AG, Fibiger HC (1990) Sexual behavior enhances central dopamine transmission in the male rat. Brain Res 530:345-348.

Pleim ET, Matochik JA, Barfield RJ, Auerbach SB (1990) Correlation of dopamine release in the nucleus accumbens with masculine sexual behavior in rats. Brain Res 524:160-163.

Rebec GV, Christensen JR, Guerra C, Bardo MT (1997) Regional and temporal differences in real-time dopamine efflux in the nucleus accumbens during free-choice novelty. Brain Res 776:61-67.

Redgrave P, Prescott TJ, Gurney K (1999) Is the short-latency dopamine response too short to signal reward error? Trends Neurosci 22:146-151.

Richfield EK, Penney JB, Young AB (1989) Anatomical and affinity state comparisons between dopamine D1 and D2 receptors in the rat central nervous system. Neuroscience 30:767-777.

Robinson DL, Phillips PE, Budygin EA, Trafton BJ, Garris PA, Wightman RM (2001) Sub-second changes in accumbal dopamine during sexual behavior in male rats. NeuroReport 12:2549-2552. 
Romo R, Schultz W (1990) Dopamine neurons of the monkey midbrain: contingencies of responses to active touch during self-initiated arm movements. J Neurophysiol 63:592-606.

Sachs BD, Barfield RJ (1976) Functional analysis of masculine copulatory behavior in the rat. In: Advances in the study of behavior (Rosenblat JS, Hinde RA, Shaw E, Beer C, eds), pp 91-154. Orlando, FL: Academic.

Salamone JD, Cousins MS, Snyder BJ (1997) Behavioral functions of nucleus accumbens dopamine: empirical and conceptual problems with the anhedonia hypothesis. Neurosci Biobehav Rev 21:341-359.

Schultz W (1998) Predictive reward signal of dopamine neurons. J Neurophysiol $80: 1-27$.

Steinfels GF, Heym J, Strecker RE, Jacobs BL (1983) Behavioral correlates of dopaminergic unit activity in freely moving cats. Brain Res 258:217-228
Suaud-Chagny MF, Dugast C, Chergui K, Msghina M, Gonon F (1995) Uptake of dopamine released by impulse flow in the rat mesolimbic and striatal systems in vivo. J Neurochem 65:2603-2611.

Westerink BH (1995) Brain microdialysis and its application for the study of animal behaviour. Behav Brain Res 70:103-124.

Wightman RM, Robinson DL (2002) Transient changes in mesolimbic dopamine and their association with "reward." J Neurochem 82:721-735.

Wightman RM, Zimmerman JB (1990) Control of dopamine extracellular concentration in rat striatum by impulse flow and uptake. Brain Res Brain Res Rev 15:135-144.

Williams GV, Millar J (1990) Differential actions of endogenous and iontophoretic dopamine in rat striatum. Eur J Neurosci 2:658-661.

Wise RA, Rompre PP (1989) Brain dopamine and reward. Annu Rev Psychol 40:191-225. 